Article

\title{
Estimates of Coefficient Functionals for Functions Convex in the Imaginary-Axis Direction
}

\author{
Paweł Zaprawa *(D) and Katarzyna Tra̧bka-Więcław \\ Faculty of Mechanical Engineering, Lublin University of Technology, ul. Nadbystrzycka 36, \\ 20-618 Lublin, Poland; k.trabka@pollub.pl \\ * Correspondence: p.zaprawa@pollub.pl
}

Received: 11 September 2020; Accepted: 19 October 2020; Published: 20 October 2020

check for updates

\begin{abstract}
Let $\mathcal{C}_{0}(h)$ be a subclass of analytic and close-to-convex functions defined in the open unit disk by the formula $\operatorname{Re}\left\{\left(1-z^{2}\right) f^{\prime}(z)\right\}>0$. In this paper, some coefficient problems for $\mathcal{C}_{0}(h)$ are considered. Some properties and bounds of several coefficient functionals for functions belonging to this class are provided. The main aim of this paper is to find estimates of the difference and of sum of successive coefficients, bounds of the sum of the first $n$ coefficients and bounds of the $n$-th coefficient. The obtained results are used to determine coefficient estimates for both functions convex in the imaginary-axis direction with real coefficients and typically real functions. Moreover, the sum of the first initial coefficients for functions with a positive real part and with a fixed second coefficient is estimated.
\end{abstract}

Keywords: close-to-convex functions; convexity in imaginary-axis direction; coefficient problems; typically real functions; successive coefficients

\section{Introduction}

Coefficient problems of analytic functions have always been of the great interest to researchers. Let $\mathcal{A}$ be a class of functions of the form

$$
f(z)=z+\sum_{n=2}^{\infty} a_{n} z^{n}
$$

which are analytic in the open unit disk $\Delta=\{z \in \mathbb{C}:|z|<1\}$. There are many papers in which the $n$-th coefficient $a_{n}$ has been estimated in various subclasses of analytic functions. The difference of the moduli of successive coefficients $\left|a_{n+1}\right|-\left|a_{n}\right|$ of a certain class of functions was also estimated (see, for example, [1-4]). The idea of estimating the difference of successive coefficients $\left|a_{n+1}-a_{n}\right|$ follows from the obvious inequality

$$
|| a_{n+1}|-| a_{n}|| \leq\left|a_{n+1}-a_{n}\right|
$$

Robertson [5] proved that $3\left|a_{n+1}-a_{n}\right| \leq(2 n+1)\left|a_{2}-1\right|$ for the class of convex functions. Some bounds of $\left|a_{n+1}-a_{n}\right|$ for convex functions satisfying an additional condition that the second coefficient is fixed were obtained by Li and Sugawa in [6]. Under this additional condition Peng and Obradović [7] found the estimates of $\left|a_{3}-a_{2}\right|$ and $\left|a_{4}-a_{3}\right|$ for a few subclasses of univalent functions.

Motivated by the idea of considering univalent functions with the second coefficient fixed, we discuss a particular subclass of the class of close-to-convex functions. In this class, we find the bounds of the difference of successive coefficients and the sum of successive coefficients. Moreover, we estimate the sum of the first $n$ coefficients, the $n$-th coefficient and the functional $(n+1) a_{n+1}-n a_{n}$. 
Let us start with the notation and the definitions. By $\mathcal{P}$ we denote the class of analytic functions $q$ with a positive real part in $\Delta$, having the Taylor series expansion

$$
q(z)=1+\sum_{n=1}^{\infty} p_{n} z^{n}
$$

A subclass of $\mathcal{P}$ consisting of functions with real coefficients is denoted by $\mathcal{P}_{\mathbb{R}}$.

Let $\mathcal{T}$ denote the class of typically real functions, i.e., functions $f \in \mathcal{A}$ which satisfy the condition $\operatorname{Im}\{z\} \cdot \operatorname{Im}\{f(z)\} \geq 0$ for all $z \in \Delta$. All coefficients of any $f \in \mathcal{T}$ are real. This results in the symmetry of $f(\Delta)$ with respect to the real axis. It is worth recalling that there exists a unique correspondence between the functions in $\mathcal{T}$ and $\mathcal{P}_{\mathbb{R}}$ (see, [8])

$$
q \in \mathcal{P}_{\mathbb{R}} \Leftrightarrow \frac{z}{1-z^{2}} q(z) \in \mathcal{T} .
$$

Let $\mathcal{S}^{*}$ denote the class of starlike functions, i.e., functions $f \in \mathcal{A}$ such that $\operatorname{Re}\left\{z f^{\prime}(z) / f(z)\right\}>0$ for all $z \in \Delta$. Given that $\beta \in(-\pi / 2, \pi / 2)$ and $g \in \mathcal{S}^{*}$, a function $f \in \mathcal{A}$ is called close-to-convex with argument $\beta$ with respect to $g$ if

$$
\operatorname{Re}\left\{\frac{e^{i \beta} z f^{\prime}(z)}{g(z)}\right\}>0, \quad z \in \Delta .
$$

The class of all functions satisfying (3) is denoted by $\mathcal{C}_{\beta}(g)$ (see [9]). Coefficient problems for the class $\mathcal{C}_{0}(k)$, where $k$ is the Koebe function $k(z)=z /(1-z)^{2}$, were discussed in a few papers (see, for example, [10-13]).

In this paper we consider the subclass $\mathcal{C}_{0}(h)$ of close-to-convex functions $\mathcal{C}_{\beta}(g)$, where $h(z)=z /\left(1-z^{2}\right)$. It follows from the definition of $\mathcal{C}_{\beta}(g)$ that

$$
f \in \mathcal{C}_{0}(h) \Leftrightarrow \operatorname{Re}\left\{\left(1-z^{2}\right) f^{\prime}(z)\right\}>0,
$$

or equivalently,

$$
f \in \mathcal{C}_{0}(h) \Leftrightarrow f^{\prime}(z)=\frac{1}{1-z^{2}} q(z)
$$

where $q$ is in $\mathcal{P}$.

Directly from the properties of $\mathcal{P}$, it follows that $\mathcal{C}_{0}(h)$ is a convex family, i.e., $\alpha f+(1-\alpha) g \in \mathcal{C}_{0}(h)$ providing that $f, g \in \mathcal{C}_{0}(h)$ and $\alpha \in[0,1]$. Moreover, the following property of symmetry is valid in $\mathcal{C}_{0}(h)$ : if $f \in \mathcal{C}_{0}(h)$, then $\tilde{f} \in \mathcal{C}_{0}(h), \tilde{f}(z)=\overline{f(\bar{z})}$. It is clear that $\tilde{f}(\Delta)$ and $f(\Delta)$ are mutually symmetric with respect to the real axis. Other important properties of $\mathcal{C}_{0}(h)$ are given in the three following theorems (see, [14]).

Theorem 1. If $f \in \mathcal{C}_{0}(h)$ is of the Form (1), then $\left|a_{n}\right| \leq 1$.

Theorem 2. If $f \in \mathcal{C}_{0}(h)$, then $f$ is convex in the direction of the imaginary axis.

Theorem 3. Let all coefficients of $f$ given by (1) be real. Then,

$$
f \in \mathcal{C}_{0}(h) \Leftrightarrow f \in \mathcal{K}_{\mathbb{R}}(i)
$$

In the above, $\mathcal{K}_{\mathbb{R}}(i)$ denotes the class of functions of the Form (1) which are convex in the direction of the imaginary axis and have all real coefficients. Robertson [15] proved that

$$
f \in \mathcal{K}_{\mathbb{R}}(i) \Leftrightarrow z f^{\prime}(z) \in \mathcal{T} .
$$


From (5), it follows that

$$
n a_{n}=p_{1}+p_{3}+\ldots+p_{n-1} \quad \text { if } n \text { is even }
$$

and

$$
n a_{n}=1+p_{2}+\ldots+p_{n-1} \text { if } n \text { is odd , }
$$

where $p_{n}$ are the coefficients of functions from the class $\mathcal{P}$.

In this paper we find bounds of different functionals depending on the second coefficient $a_{2}$ of $f \in \mathcal{C}_{0}(h)$. In fact, it is more convenient to express our results in terms of $p=p_{1}$, applying the correspondence

$$
2 a_{2}=p
$$

To make the results more legible, we define the class $\mathcal{C}_{0}(h, p), p \in[-2,2]$ as follows

$$
\mathcal{C}_{0}(h, p)=\left\{f \in \mathcal{C}_{0}(h): f^{\prime \prime}(0)=p\right\}
$$

Clearly,

$$
\bigcup_{-2 \leq p \leq 2} \mathcal{C}_{0}(h, p) \subset \mathcal{C}_{0}(h) \text { and } \bigcup_{-2 \leq p \leq 2} \mathcal{C}_{0}(h, p) \neq \mathcal{C}_{0}(h) .
$$

Observe that in two particular cases: when $p=-2$ or $p=2$, the class $\mathcal{C}_{0}(h, p)$ consists of only one function. Namely, if $p=-2$, then $q(z)=(1-z) /(1+z) \in \mathcal{P}$; so $f(z)=z /(1+z)$ is the only member of $\mathcal{C}_{0}(h,-2)$. If $p=2$, then $q(z)=(1+z) /(1-z) \in \mathcal{P}$ and $f(z)=z /(1-z)$ is the only member of $\mathcal{C}_{0}(h, 2)$.

For $f \in \mathcal{A}$ given by (1) we define

$$
\begin{gathered}
F_{n}=a_{n+1}-a_{n} \\
G_{n}=(n+1) a_{n+1}-n a_{n}, \\
S_{n}=1+a_{2}+a_{3}+\ldots+a_{n} .
\end{gathered}
$$

Moreover, for $q \in \mathcal{P}$ given by (2) we define

$$
\begin{gathered}
P_{n}=p_{1}+p_{2}+\ldots+p_{n} \\
Q_{n}=p_{1}-p_{2}+\ldots+(-1)^{n+1} p_{n} .
\end{gathered}
$$

\section{Auxiliary Lemmas}

In order to prove our results, we need a few lemmas concerning functions in the class $\mathcal{P}$. The first one is known as Caratheodory's lemma (see, for example, [16]). The second one is due to Hayami and Owa ([17]) and the third one is the result of Libera and Złotkiewicz $([18,19])$.

Lemma 1 ([16]). If $q \in \mathcal{P}$ is given by (2), then the sharp inequality $\left|p_{n}\right| \leq 2$ holds for $n \geq 1$.

Lemma 2 ([17]). If $q \in \mathcal{P}$ and $\mu \in \mathbb{R}$, then the sharp estimate

$$
\left|p_{n}-\mu p_{k} p_{n-k}\right| \leq \max \{2,2|2 \mu-1|\}
$$

holds for $n, k=1,2, \ldots, n>k$.

Lemma $3([18,19])$. If $q \in \mathcal{P}$, then

(i) $2 p_{2}=p_{1}^{2}+x\left(4-p_{1}^{2}\right)$ ，

(ii) $4 p_{3}=p_{1}^{3}+2 p_{1}\left(4-p_{1}^{2}\right) x-p_{1}\left(4-p_{1}^{2}\right) x^{2}+2\left(4-p_{1}^{2}\right)\left(1-|x|^{2}\right) y$, 
for some $x$ and $y$ such that $|x| \leq 1,|y| \leq 1$.

If $x=1$, then

$$
\begin{aligned}
q(z) & =\frac{1}{2}\left(1+\frac{p}{2}\right) \frac{1+z}{1-z}+\frac{1}{2}\left(1-\frac{p}{2}\right) \frac{1-z}{1+z} \\
& =1+p z+2 z^{2}+p z^{3}+2 z^{4}+\ldots
\end{aligned}
$$

If $x=-1$, then

$$
q(z)=\frac{1-z^{2}}{1-p z+z^{2}}=1+p z+\left(p^{2}-2\right) z^{2}+\left(p^{3}-3 p\right) z^{3}+\ldots
$$

The following lemmas were proved by Brown (Theorem 2.1 with $m=1$ and $v=\pi$ in [20]) and Lecko (Corollary 2.3 in [21]).

Lemma 4 ([20]). If $q \in \mathcal{P}$ is given by (2), then

$$
\left|p_{n+1}+p_{n}\right| \leq 2 \sqrt{2+\operatorname{Re}\left\{p_{1}\right\}}
$$

and

$$
\left|p_{n+1}-p_{n}\right| \leq 2 \sqrt{2-\operatorname{Re}\left\{p_{1}\right\}} .
$$

Lemma 5 ([21]). If $q \in \mathcal{P}$ is given by (2), then

$$
\left|p_{2}+2 p_{1}+2\right| \leq 2\left(2+\operatorname{Re}\left\{p_{1}\right\}\right)
$$

and

$$
\left|p_{n+1}+2 p_{n}+p_{n-1}\right| \leq 2\left(2+\operatorname{Re}\left\{p_{1}\right\}\right) .
$$

The next two lemmas relate to the bounds of functionals $P_{n}$ and $Q_{n}$ defined in (12) and (13).

Lemma 6. If $q \in \mathcal{P}$ is given by (2) and $p=p_{1} \in \mathbb{R}$, then

$$
\left|P_{n}\right| \leq\left\{\begin{array}{ll}
n+1+\frac{1}{2}(n-1) p & , p \in[-2,-2 / 3] \\
n+\frac{1}{2}(n-4) p & , p \in[-2 / 3,0] \\
n+\frac{1}{2} n p & , p \in[0,2]
\end{array} \quad \text { if } n\right. \text { is even }
$$

and

$$
\left|P_{n}\right| \leq\left\{\begin{array}{ll}
n+\frac{1}{2}(n-2) p & , p \in[-2,0] \\
n+\frac{1}{2} n p & , p \in[0,2]
\end{array} \quad \text { if } n \text { is odd } .\right.
$$

Equality holds for Function (14), if $n$ is even and $p \in[0,2]$, and if $p=-2$ and $p=2$ for all positive integers $n$.

Proof. Let $n$ be even. We can write

$$
2 P_{n}=\sum_{j=1}^{\frac{n}{2}}\left(p_{2 j-1}+2 p_{2 j}+p_{2 j+1}\right)+p_{1}-p_{n+1}
$$

or

$$
2 P_{n}=\sum_{j=1}^{\frac{n}{2}-1}\left(p_{2 j}+2 p_{2 j+1}+p_{2 j+2}\right)+2 p_{1}+p_{2}+p_{n} .
$$


Applying Formula (17) we obtain

$$
2\left|P_{n}\right| \leq n(2+p)+|p|+2
$$

or

$$
2\left|P_{n}\right| \leq(n-2)(2+p)+2|p|+4
$$

which results in

$$
\left|P_{n}\right| \leq \min \left\{\frac{1}{2}[n(2+p)+|p|+2] ; \frac{1}{2}[(n-2)(2+p)+2|p|+4]\right\} .
$$

This proves (18).

Let $n$ be odd. Then

$$
2 P_{n}=\sum_{j=1}^{\frac{n}{2}-1}\left(p_{2 j-1}+2 p_{2 j}+p_{2 j+1}\right)+p_{1}+p_{n}
$$

so

$$
2\left|P_{n}\right| \leq(n-2)(2+p)+|p|+2,
$$

which is equivalent to (19).

It is easy to check that $P_{n}=n+\frac{1}{2} n p$, if $h$ is given by (14), $n$ is even and $p \in[0,2]$. Equalities for the cases $p=-2$ and $p=2$ are also easy to verify.

If we take $q(-z)$ instead of $q(z)$ in Lemma 6, then we obtain the estimate of $Q_{n}$.

Lemma 7. If $q \in \mathcal{P}$ is given by (2) and $p=p_{1} \in \mathbb{R}$, then

$$
\left|Q_{n}\right| \leq\left\{\begin{array}{ll}
n-\frac{1}{2} n p & , p \in[-2,0] \\
n-\frac{1}{2}(n-4) p & , p \in[0,2 / 3] \\
n+1-\frac{1}{2}(n-1) p & , p \in[2 / 3,2]
\end{array} \quad \text { if } n\right. \text { is even }
$$

and

$$
\left|Q_{n}\right| \leq\left\{\begin{array}{ll}
n-\frac{1}{2} n p & , p \in[-2,0] \\
n-\frac{1}{2}(n-2) p & , p \in[0,2]
\end{array} \quad \text { if } n \text { is odd } .\right.
$$

Equality holds for Function (14), if $n$ is even and $p \in[-2,0]$ and if $p=-2$ and $p=2$ for all positive integers $n$.

The last lemma is a special case of a more general result due to Choi, Kim and Sugawa [22]. Let $\bar{\Delta}=\{z \in \mathbb{C}:|z| \leq 1\}$. Define

$$
Y(a, b, c)=\max _{z \in \bar{\Delta}}\left(\left|a+b z+c z^{2}\right|+1-|z|^{2}\right), \quad a, b, c \in \mathbb{R} .
$$

Lemma 8. If $a c<0$, then

$$
Y(a, b, c)= \begin{cases}1+|a|+\frac{b^{2}}{4(1+|c|)}, & |b|<2(1+|c|) \text { and } b^{2}<-4 a\left(1-c^{2}\right) / c \\ 1-|a|+\frac{b^{2}}{4(1-|c|)}, & |b|<2(1-|c|) \text { and } b^{2} \geq-4 a\left(1-c^{2}\right) / c \\ R(a, b, c), & \text { otherwise, }\end{cases}
$$


where

$$
R(a, b, c)= \begin{cases}|a|+|b|-|c|, & |a b| \geq|c|(|b|+4|a|) \\ -|a|+|b|+|c|, & |a b| \leq|c|(|b|-4|a|) \\ (|c|+|a|) \sqrt{1-\frac{b^{2}}{4 a c}}, & \text { otherwise. }\end{cases}
$$

If $a c \geq 0$, then

$$
Y(a, b, c)= \begin{cases}|a|+|b|+|c|, & |b| \geq 2(1-|c|), \\ 1+|a|+\frac{b^{2}}{4(1-|c|)}, & |b|<2(1-|c|) .\end{cases}
$$

\section{Bounds of $\left|F_{n}\right|$}

In this section we estimate the difference of successive coefficients for $f \in \mathcal{C}_{0}(h, p)$. The functional $F_{n}$ is defined in (9).

Theorem 4. If $f \in \mathcal{C}_{0}(h, p), p \in[-2,2]$, then

$$
\left|F_{n}\right| \leq \begin{cases}\frac{1}{n+1}(2+n \sqrt{2-p}), & \text { if } n \text { is even, } \\ \frac{1}{n+1}(|p-1|+1+(n-1) \sqrt{2-p}), & \text { if } n \text { is odd. }\end{cases}
$$

Equality holds for $f(z)=z /(1+z)$, if $p=-2$.

Proof. If $n=2 k, k \in \mathbb{N}$, then from (7) we have:

$$
\begin{aligned}
\left|F_{2 k}\right| & =\left|a_{2 k+1}-a_{2 k}\right| \\
& =\left|\frac{1}{2 k+1}\left(1+p_{2}+p_{4}+\cdots+p_{2 k}\right)-\frac{1}{2 k}\left(p_{1}+p_{3}+\cdots+p_{2 k-1}\right)\right| \\
& \leq \frac{1}{2 k+1}+\frac{1}{2 k+1}\left(\left|p_{2}-p_{1}\right|+\cdots+\left|p_{2 k}-p_{2 k-1}\right|\right) \\
& +\frac{1}{2 k(2 k+1)}\left|p_{1}+p_{3}+\cdots+p_{2 k-1}\right| .
\end{aligned}
$$

Taking into account Lemmas 1 and 4, we obtain

$$
\begin{aligned}
\left|F_{2 k}\right| & \leq \frac{1}{2 k+1}+\frac{1}{2 k+1} \cdot k \cdot 2 \sqrt{2-p}+\frac{1}{2 k(2 k+1)} \cdot 2 k \\
& =\frac{1}{2 k+1}(2+2 k \sqrt{2-p})
\end{aligned}
$$

If $n=2 k+1, k \in \mathbb{N}$, then from (8) we have:

$$
\begin{aligned}
\left|F_{2 k+1}\right| & =\left|a_{2 k+2}-a_{2 k+1}\right| \\
& =\left|\frac{1}{2 k+2}\left(p_{1}+p_{3}+\cdots+p_{2 k+1}\right)-\frac{1}{2 k+1}\left(1+p_{2}+p_{4}+\cdots+p_{2 k}\right)\right| \\
& \leq \frac{1}{2 k+2}\left(\left|p_{1}-1\right|+\left|p_{3}-p_{2}\right|+\cdots+\left|p_{2 k+1}-p_{2 k}\right|\right) \\
& +\frac{1}{(2 k+2)(2 k+1)}\left|1+p_{2}+p_{4}+\cdots+p_{2 k}\right| .
\end{aligned}
$$


Taking into account Lemmas 1 and 4, we obtain

$$
\begin{aligned}
\left|F_{2 k+1}\right| & \leq \frac{1}{2 k+2}(|p-1|+k \cdot 2 \sqrt{2-p})+\frac{1}{(2 k+2)(2 k+1)} \cdot(1+2 k) \\
& =\frac{1}{2 k+2}(|p-1|+1+2 k \sqrt{2-p})
\end{aligned}
$$

If $p=-2$, then $f(z)=z /(1+z)$. In this case $\left|F_{n}\right|=2$. Hence, (22) is sharp for $p=-2$.

In the following theorem we derive the sharp bounds of $\left|F_{2}\right|$ and $\left|F_{3}\right|$ for $f \in \mathcal{C}_{0}(h, p)$ and each $p \in[-2,2]$.

Theorem 5. If $f \in \mathcal{C}_{0}(h, p), p \in[-2,2]$, then the following sharp inequalities hold

$$
\left|F_{2}\right| \leq \begin{cases}\frac{1}{2}(2-p) & , p \in[-2,1] \\ \frac{1}{6}(2-p)(2 p+1) & , p \in[1,2]\end{cases}
$$

and

$$
\left|F_{3}\right| \leq \begin{cases}\frac{1}{36}(38-17 p) & , p \in[-2,5 / 3] \\ \frac{1}{12}(2-p)\left(3 p^{2}+2 p-2\right) & , p \in[5 / 3,2]\end{cases}
$$

Proof. From (7) and (8) we obtain

$$
F_{2}=\frac{1}{6}\left(2 p_{2}-3 p_{1}+2\right) \quad \text { and } \quad F_{3}=\frac{1}{12}\left(3 p_{3}-4 p_{2}+3 p_{1}-4\right)
$$

Application of Lemma 3 leads to

$$
6\left|F_{2}\right|=|2-p||1-p+(2+p) x| \leq \begin{cases}3(2-p) & , p \in[-2,1] \\ (2-p)(2 p+1) & , p \in[1,2]\end{cases}
$$

and

$$
12\left|F_{3}\right|=\left|\frac{1}{4}(p-2)\left(3 p^{2}-2 p+8\right)+\left(4-p^{2}\right)\left(\frac{3}{2} p-2\right) x-\frac{3}{4}\left(4-p^{2}\right) p x^{2}+\frac{3}{2}\left(4-p^{2}\right)\left(1-|x|^{2}\right) y\right| .
$$

If $p=2$ or $p=-2$, we immediately obtain $\left|F_{3}\right| \leq 0$ or $\left|F_{3}\right| \leq 2$, respectively. Now, we assume that $p \in(-2,2)$. For $F_{3}$ we have

$$
12\left|F_{3}\right| \leq \frac{3}{2}\left(4-p^{2}\right)\left[\left|a+b x+c x^{2}\right|+1-|x|^{2}\right],
$$

with

$$
a=-\frac{3 p^{2}-2 p+8}{6(p+2)}, \quad b=p-\frac{4}{3}, \quad c=-\frac{1}{2} p .
$$

We use Lemma 8. For $p \in(-2,0)$ we have:

$$
a c<0 \text { and }|b|<2(1+|c|) \text { and } b^{2}<-4 a\left(1-c^{2}\right) / c
$$

Thus, for $p \in(-2,0)$ we obtain

$$
12\left|F_{3}\right| \leq \frac{3}{2}\left(4-p^{2}\right)\left(1+\left|\frac{3 p^{2}-2 p+8}{6(p+2)}\right|+\frac{\left(p-\frac{4}{3}\right)^{2}}{4\left(1+\left|-\frac{1}{2} p\right|\right)}\right)=\frac{1}{3}(38-17 p) .
$$


Clearly, $a c \geq 0$ for $p \in[0,2)$. Then the inequality $|b| \geq 2(1-|c|)$ holds for $p \in[5 / 3,2)$. So, for $p \in[5 / 3,2)$ we have

$$
12\left|F_{3}\right| \leq \frac{3}{2}\left(4-p^{2}\right)\left(\left|\frac{3 p^{2}-2 p+8}{6(p+2)}\right|+\left|p-\frac{4}{3}\right|+\left|-\frac{1}{2} p\right|\right)=(2-p)\left(3 p^{2}+2 p-2\right) .
$$

For $p \in[0,5 / 3]$ we obtain

$$
12\left|F_{3}\right| \leq \frac{3}{2}\left(4-p^{2}\right)\left(1+\left|\frac{3 p^{2}-2 p+8}{6(p+2)}\right|+\frac{\left(p-\frac{4}{3}\right)^{2}}{4\left(1-\left|-\frac{1}{2} p\right|\right)}\right)=\frac{1}{3}(38-17 p) .
$$

The sharpness of the bounds follows from Lemmas 3 and 8. From Lemma 3 we deduce that the equality in the estimate of $F_{2}$ holds if $x=1$ or $x=-1$, so for $q$ of the Form (14) or (15). In the first case the corresponding function $f \in \mathcal{C}_{0}(h, p)$ is such that

$$
f^{\prime}(z)=\frac{1}{2}\left(1+\frac{p}{2}\right) \frac{1}{(1-z)^{2}}+\frac{1}{2}\left(1-\frac{p}{2}\right) \frac{1}{(1+z)^{2}},
$$

so

$$
f(z)=\frac{z+\frac{p}{2} z^{2}}{1-z^{2}}=z+\frac{p}{2} z^{2}+z^{3}+\frac{p}{2} z^{4}+\ldots
$$

In the other case

$$
f^{\prime}(z)=\frac{1}{1-p z+z^{2}}
$$

so

$$
f(z)=\frac{2}{\sqrt{4-p^{2}}} \arctan \left(\frac{z \sqrt{4-p^{2}}}{2-p z}\right)=z+\frac{p}{2} z^{2}+\frac{1}{3}\left(p^{2}-1\right) z^{3}+\frac{p}{4}\left(p^{2}-2\right) z^{4}+\ldots
$$

In both cases, if $p=2$ or $p=-2$, then $f(z)=z /(1-z)$ and $f(z)=z /(1+z)$, respectively.

It is easy to observe that the equality in (24) for $p \in[-5 / 3,2]$ holds for the function given by (26).

\section{Bounds of $\left|G_{n}\right|$}

In this section, we find estimates of the functional $G_{n}$ defined in (10). If $f \in \mathcal{C}_{0}(h, p)$, then from (7) and (8) it follows that

$$
G_{n}=(-1)^{n}\left(1-Q_{n}\right)
$$

From (27) we obtain that

$$
\left|G_{n}\right| \leq 1+\left|Q_{n}\right|
$$

This result can be improved for even $n$, if we rearrange $G_{n}$ as follows

$$
2 G_{n}=\left(2-2 p_{1}+p_{2}\right)+\sum_{j=1}^{\frac{n}{2}-1}\left(p_{2 j}-2 p_{2 j+1}+p_{2 j+2}\right)+p_{n} .
$$

Hence, from Formulas (16) and (17),

$$
2\left|G_{n}\right| \leq n(2-p)+2
$$

Combining (28) and Lemma 7, we can formulate the main theorem of this section.

Theorem 6. If $f \in \mathcal{C}_{0}(h, p), p \in[-2,2]$, then

$$
\left|G_{n}\right| \leq n+1-\frac{1}{2} n p \quad \text { if } n \text { is even }
$$


and

$$
\left|G_{n}\right| \leq\left\{\begin{array}{ll}
n+1-\frac{1}{2} n p & , p \in[-2,0] \\
n+1-\frac{1}{2}(n-2) p & , p \in[0,2]
\end{array} \quad \text { if } n \text { is odd } .\right.
$$

For each $p \in[-2,2]$ and even $n$, equality holds for Function (25). Moreover, for $p=-2$ and all positive integers $n$, equalities hold for $f(z)=z /(1+z)$.

Apart of the sharp bounds of $\left|G_{n}\right|$ for even $n$, we can also derive the sharp bound of $G_{3}$.

Theorem 7. If $f \in \mathcal{C}_{0}(h, p), p \in[-2,2]$, then the following sharp inequality holds

$$
\left|G_{3}\right| \leq \begin{cases}\frac{7}{4}(2-p) & , p \in[-2,4 / 3] \\ \frac{1}{4}(6-p) & , p \in[4 / 3,2]\end{cases}
$$

Proof. From (7) and (8),

$$
G_{3}=p_{3}-p_{2}+p-1
$$

The application of Lemma 3 leads to

$$
\left|G_{3}\right|=\frac{1}{4}\left|p^{3}-2 p^{2}+4 p-4+2\left(4-p^{2}\right)(p-1) x-\left(4-p^{2}\right) p x^{2}+2\left(4-p^{2}\right)\left(1-|x|^{2}\right) y\right| .
$$

If $p=2$ or $p=-2$, we immediately obtain $\left|G_{3}\right| \leq 1$ or $\left|G_{3}\right| \leq 7$, respectively. Now, we assume that $p \in(-2,2)$. For $G_{3}$ we have

$$
\left|G_{3}\right| \leq \frac{1}{2}\left(4-p^{2}\right)\left[\left|a+b x+c x^{2}\right|+1-|x|^{2}\right]
$$

with

$$
a=\frac{p^{3}-2 p^{2}+4 p-4}{2\left(4-p^{2}\right)}, \quad b=p-1, \quad c=-\frac{1}{2} p .
$$

Let $p_{*}=1.295 \ldots$ be the only solution of $p^{3}-2 p^{2}+4 p-4=0$ in $(-2,2)$. For $p \in\left[0, p_{*}\right]$ we have $a c \geq 0$ and $|b|<2(1-|c|)$, so

$$
\left|G_{3}\right| \leq \frac{1}{4}\left[2\left(4-p^{2}\right)-\left(p^{3}-2 p^{2}+4 p-4\right)+(2+p)(p-1)^{2}\right] .
$$

If $p \in(-2,0) \cup\left(p_{*}, 2\right)$, then $a c<0$. For such $p$ we discuss three cases.

Let $p \in(-2,0)$. Then, $b^{2}<-4 a\left(1-c^{2}\right) / c$ is equivalent to

$$
p(p-1)^{2}>p^{3}-2 p^{2}+4 p-4,
$$

therefore, this condition as well as $|b|<2(1+|c|)$ hold for all $p \in(-2,0)$. In this case, from Lemma 8 we obtain the bound given in (32).

Similarly, for positive $p$, the condition $b^{2}<-4 a\left(1-c^{2}\right) / c$ is equivalent to

$$
p(p-1)^{2}<p^{3}-2 p^{2}+4 p-4 .
$$

This means that $p>4 / 3$. For such $p$ the condition $|b|<2(1+|c|)$ is also satisfied. Once again we obtain (32).

Finally, if $p \in\left(p_{*}, 4 / 3\right]$, then $b^{2} \geq-4 a\left(1-c^{2}\right) / c$. Additionally, $|b|<2(1-|c|)$. This results in

$$
\left|G_{3}\right| \leq \frac{1}{4}\left[2\left(4-p^{2}\right)+\left(p^{3}-2 p^{2}+4 p-4\right)+(2-p)(p-1)^{2}\right]=\frac{1}{4}(6-p) .
$$

The sharpness of the bounds follows from Lemmas 3 and 8. 


\section{Bounds of $\left|S_{n}\right|$}

In this section we determine the bounds of the functional $S_{n}$ defined in (11), i.e., we find estimates of the sum of the first $n$ coefficients of $f \in \mathcal{C}_{0}(h, p)$. To prove the main theorem of this section, we use the following three theorems. The proof of the first one is analogous to the proof of Theorem 4.

Theorem 8. If $f \in \mathcal{C}_{0}(h, p), p \in[-2,2]$, then

$$
\left|a_{n}+a_{n+1}\right| \leq \begin{cases}\frac{1}{n+1}(2+n \sqrt{2+p}), & \text { if } n \text { is even } \\ \frac{1}{n+1}(|p+1|+1+(n-1) \sqrt{2+p}), & \text { if } n \text { is odd }\end{cases}
$$

For $p=2$ we have $\left|a_{n}+a_{n+1}\right| \leq 2$.

From (7) we obtain that, if $f \in \mathcal{C}_{0}(h, p), p \in[-2,2]$, then

$$
\left|S_{2}\right| \leq 1+\frac{1}{2} p .
$$

Theorem 9. If $f \in \mathcal{C}_{0}(h, p), p \in[-2,2]$, then the following sharp inequality holds

$$
\left|S_{3}\right| \leq 2+\frac{1}{2} p .
$$

Proof. From (7) and (8) we obtain

$$
S_{3}=\frac{1}{6}\left(8+3 p_{1}+2 p_{2}\right) .
$$

The application of Lemma 3 leads to

$$
6\left|S_{3}\right|=\left|8+3 p+p^{2}+\left(4-p^{2}\right) x\right| \leq 3 p+12 .
$$

The estimates (33) and (34) are sharp. Both equalities hold for the function given by (25).

Theorem 10. If $f \in \mathcal{C}_{0}(h, p), p \in[-2,2]$, then the following sharp inequality holds

$$
\left|S_{4}\right| \leq \begin{cases}\frac{1}{12}(2+p)\left(3 p^{2}-2 p+4\right) & , p \in[-2,-5 / 3] \\ \frac{1}{36}(74+35 p) & , p \in[-5 / 3,2] .\end{cases}
$$

Proof. From (7) and (8) we obtain

$$
S_{4}=\frac{1}{12}\left(16+9 p_{1}+4 p_{2}+3 p_{3}\right) .
$$

The application of Lemma 3 leads to

$$
\begin{aligned}
12\left|S_{4}\right| & =\mid 16+9 p+2 p^{2}+2\left(4-p^{2}\right) x+\frac{3}{4} p^{3}+\frac{3}{2} p\left(4-p^{2}\right) x \\
& -\frac{3}{4} p\left(4-p^{2}\right) x^{2}+\frac{3}{2}\left(4-p^{2}\right)\left(1-|x|^{2}\right) y \mid .
\end{aligned}
$$

If $p=2$ or $p=-2$, we immediately obtain $\left|S_{4}\right| \leq 4$ or $\left|S_{4}\right| \leq 0$, respectively. Now, we assume that $p \in(-2,2)$. We have

$$
12\left|S_{4}\right| \leq \frac{3}{2}\left(4-p^{2}\right)\left[\left|a+b x+c x^{2}\right|+1-|x|^{2}\right],
$$


with

$$
a=\frac{3 p^{2}+2 p+32}{6(2-p)}, \quad b=p+\frac{4}{3}, \quad c=-\frac{1}{2} p .
$$

We use Lemma 8. For $p \in(0,2)$ we have:

$$
a c<0 \text { and }|b|<2(1+|c|) \text { and } b^{2}<-4 a\left(1-c^{2}\right) / c .
$$

Thus, for $p \in(0,2)$, we obtain

$$
12\left|S_{4}\right| \leq \frac{3}{2}\left(4-p^{2}\right)\left(1+\left|\frac{3 p^{2}+2 p+32}{6(2-p)}\right|+\frac{\left(p+\frac{4}{3}\right)^{2}}{4\left(1+\left|-\frac{1}{2} p\right|\right)}\right)=\frac{1}{3}(74+35 p) .
$$

Clearly, $a c \geq 0$ for $p \in(-2,0]$. Then the inequality $|b| \geq 2(1-|c|)$ holds for $p \in(-2,-5 / 3]$. So, for $p \in(-2,-5 / 3]$ we have

$$
12\left|S_{4}\right| \leq \frac{3}{2}\left(4-p^{2}\right)\left(\left|\frac{3 p^{2}+2 p+32}{6(2-p)}\right|+\left|p+\frac{4}{3}\right|+\left|-\frac{1}{2} p\right|\right)=(2+p)\left(3 p^{2}-2 p+4\right) .
$$

For $p \in(-5 / 3,0]$ we obtain

$$
12\left|S_{4}\right| \leq \frac{3}{2}\left(4-p^{2}\right)\left(1+\left|\frac{3 p^{2}+2 p+32}{6(2-p)}\right|+\frac{\left(p+\frac{4}{3}\right)^{2}}{4\left(1-\left|-\frac{1}{2} p\right|\right)}\right)=\frac{1}{3}(74+35 p) .
$$

The sharpness of the bounds follows from Lemmas 3 and 8. It is easy to check that the equality in the estimate of $S_{4}$ for $p \in[-2,-5 / 3]$ holds for $f$ given by (26).

Now, we can state the main theorem of this section.

Theorem 11. If $f \in \mathcal{C}_{0}(h, p)$, then

(i) for odd numbers $n, n \geq 5$ and $p \in[-2,2]$ we have

$$
\left|S_{n}\right| \leq(2-\sqrt{2+p})\left(H_{n}-\frac{1}{2} H_{\frac{n-1}{2}}\right)+\frac{3 p-4}{6}+\frac{3 n-1}{6} \sqrt{2+p},
$$

(ii) for even numbers $n, n \geq 6$ and $p \in[-2,-5 / 3]$ we have

$$
\left|S_{n}\right| \leq \frac{1}{12}(p+1)\left(3 p^{2}+p+8\right)-\left(\frac{1}{2} p+\sqrt{2+p}\right) H_{\frac{n}{2}}+\frac{n-1}{2} \sqrt{2+p},
$$

(iii) for even numbers $n, n \geq 6$ and $p \in[-5 / 3,-1]$ we have

$$
\left|S_{n}\right| \leq \frac{1}{18}(37+31 p)-\left(\frac{1}{2} p+\sqrt{2+p}\right) H_{\frac{n}{2}}+\frac{n-1}{2} \sqrt{2+p},
$$

(iv) for even numbers $n, n \geq 6$ and $p \in[-1,2]$ we have

$$
\left|S_{n}\right| \leq \frac{1}{36}(74+35 p)+\sqrt{2+p}\left[\left(\frac{1}{2} \sqrt{2+p}-1\right) H_{\frac{n}{2}}+\frac{n-1}{2}-\frac{3}{4} \sqrt{2+p}\right],
$$

where $H_{n}=1+\frac{1}{2}+\frac{1}{3}+\cdots+\frac{1}{n}$. Equality holds for $f(z)=z /(1-z)$, if $p=2$.

Proof. If $n$ is odd, $n \geq 5$, then from (11) we have

$$
\left|S_{n}\right| \leq\left|S_{3}\right|+\sum_{j=2}^{\frac{n-1}{2}}\left|a_{2 j}+a_{2 j+1}\right| .
$$


Taking into account Theorems 8 and 9 , for $n \geq 5$ we get

$$
\begin{aligned}
\left|S_{n}\right| & \leq\left|S_{3}\right|+\sum_{j=2}^{\frac{n-1}{2}} \frac{2}{2 j+1}+\sqrt{2+p} \sum_{j=2}^{\frac{n-1}{2}} \frac{2 j}{2 j+1} \\
& =2+\frac{1}{2} p+2\left(H_{n}-\frac{1}{2} H_{\frac{n-1}{2}}-\frac{4}{3}\right)+\left(\frac{3 n-1}{6}-H_{n}+\frac{1}{2} H_{\frac{n-1}{2}}\right) \sqrt{2+p} \\
& =(2-\sqrt{2+p})\left(H_{n}-\frac{1}{2} H_{\frac{n-1}{2}}\right)+\frac{3 p-4}{6}+\frac{3 n-1}{6} \sqrt{2+p} .
\end{aligned}
$$

If $n$ is even, $n \geq 6$, then from (11) we have

$$
\left|S_{n}\right| \leq\left|S_{4}\right|+\sum_{j=3}^{\frac{n}{2}}\left|a_{2 j-1}+a_{2 j}\right| .
$$

Taking into account Theorem 8 , we obtain

$$
\begin{aligned}
\left|S_{n}\right| & \leq\left|S_{4}\right|+\frac{1}{2}(|p+1|+1) \sum_{j=3}^{\frac{n}{2}} \frac{1}{j}+\sqrt{2+p} \sum_{j=3}^{\frac{n}{2}} \frac{j-1}{j} \\
& =\left|S_{4}\right|+\frac{1}{2}(|p+1|+1)\left(H_{\frac{n}{2}}-\frac{3}{2}\right)+\sqrt{2+p}\left(\frac{n-1}{2}-H_{\frac{n}{2}}\right) \\
& =\left|S_{4}\right|+\left[\frac{1}{2}(|p+1|+1)-\sqrt{2+p}\right] H_{\frac{n}{2}}-\frac{3}{4}(|p+1|+1)+\frac{n-1}{2} \sqrt{2+p} .
\end{aligned}
$$

By applying Theorem 10 and making a simple calculation, we obtain the desired estimate of $S_{n}$ for even $n$.

Taking $p=2$ in Theorem 11, we obtain the sharp bound $\left|S_{n}\right| \leq n$. The sharpness of this result is a simple consequence of the sharpness of Theorems 8-10.

\section{Bounds of $\left|a_{n}\right|$}

In all results presented above, the estimates of the functionals defined for functions $f \in \mathcal{C}_{0}(h)$ depend on the fixed second coefficient. Consequently, the natural question arises about the bound of the $n$-th coefficient.

Observe that the function $f$ given by (1) has all even coefficients equal to 1 , independently the second coefficient. Therefore, we may pose a question about the bounds of odd coefficients when $a_{2}$ is fixed or the bounds of all coefficients under the assumption that $a_{3}$ is fixed. We shall give the answer to the second question provided that $a_{3}$ is a real number.

We need the lemma which is a simple consequence of the set of variability of $\left(p_{1}, p_{2}\right)$, where $p_{1}$ and $p_{2}$ are the coefficients of a function $q \in \mathcal{P}_{\mathbb{R}}$.

Lemma 9. If $q \in \mathcal{P}_{\mathbb{R}}$, then $p_{1}^{2}-2 \leq p_{2} \leq 2$.

In view of this lemma, we immediately get that, if $f \in \mathcal{C}_{0}(h)$ and $a=a_{3} \in \mathbb{R}$, then $a \in[-1 / 3,1]$. Now, we are ready to derive the bound of $a_{n}$ for $f \in \mathcal{C}_{0}(h)$.

Theorem 12. If $f \in \mathcal{C}_{0}(h)$ and $a=a_{3} \in \mathbb{R}$, then

$$
\left|a_{2 k}\right| \leq \begin{cases}\frac{1}{2} \sqrt{3 a+1} & \text { if } k \text { is even } \\ \frac{1}{2}\left(1-\frac{1}{k}\right) \sqrt{3 a+1}+\frac{1}{k} & \text { if } k \text { is odd }\end{cases}
$$

and

$$
\left|a_{2 k+1}\right| \leq \begin{cases}\frac{1}{2 k+1}(1+k \sqrt{3 a+1}) & \text { if } k \text { is even } \\ \frac{1}{2 k+1}[3|a|+(k-1) \sqrt{3 a+1}] & \text { if } k \text { is odd }\end{cases}
$$


Observe that these estimates are sharp if $a=1$, which means that $p_{2}=2$. The above four cases produce $\left|a_{n}\right| \leq 1$, which clearly holds for $f(z)=z /(1-z)$. In this case, the corresponding function $q$ in the class $\mathcal{P}$ is of the form $q(z)=(1+z) /(1-z)$. Moreover, the bounds from Theorem 12 are also sharp for $a=-1 / 3$ if $n=2 k+1$ and if $n=2 k$ with even $k$. If $a=-1 / 3$, then $p_{2}=-2$. In this case, by Lemma $9, p_{1}=0$. Hence, $q(z)=\left(1-z^{2}\right) /\left(1+z^{2}\right)$ and the corresponding function $f \in \mathcal{C}_{0}(h)$ is of the form

$$
f(z)=\arctan (z)=\sum_{j=0}^{\infty} \frac{(-1)^{j}}{2 j+1} z^{2 j+1},
$$

for which

$$
a_{2 k}=0 \quad \text { and } \quad a_{2 k+1}=\frac{(-1)^{k}}{2 k+1} .
$$

Proof of Theorem 12. Let $n=2 k+1$. From (8) it follows that

$$
(2 k+1)\left|a_{2 k+1}\right| \leq \begin{cases}1+\left|p_{2}+p_{4}\right|+\left|p_{6}+p_{8}\right|+\ldots+\left|p_{2 k-2}+p_{2 k}\right| & \text { for odd } k \\ \left|1+p_{2}\right|+\left|p_{4}+p_{6}\right|+\ldots+\left|p_{2 k-2}+p_{2 k}\right| & \text { for even } k .\end{cases}
$$

Now, it is enough to apply Lemma 4 (in its general version) and the relation $3 a=1+p_{2}$.

For even $n$ the proof is similar.

\section{Applications for Typically Real Functions}

From Theorem 3 it follows that $\mathcal{K}_{\mathbb{R}}(i) \subset \mathcal{C}_{0}(h)$. Therefore, all estimates obtained in Sections 3-6 are also valid for $\mathcal{K}_{\mathbb{R}}(i)$. These bounds are sharp for any fixed $p$ in $[-2,2]$ and for $n=2$ (Formulae (23), (29) and (33)), for $n=3$ (Formulae (24), (31) and (34)) and for $n=4$ (Formula (35)). One of the results, namely (29), is sharp even for all positive even integers $n$. In the majority of cases, the extremal functions are those given by (25) and by (26).

The application of the relation between $\mathcal{K}_{\mathbb{R}}(i)$ and $\mathcal{T}$ and the results found in Section 4 lead to obtaining the estimates of successive coefficients for typically real functions. These results would be difficult to obtain in any other way.

Let $g(z)=z f^{\prime}(z)$. From (6), we know that $f \in \mathcal{K}_{\mathbb{R}}(i)$, if and only if, $g \in \mathcal{T}$. If $f$ is of the Form (1) and

$$
g(z)=z+b_{2} z^{2}+\ldots
$$

then

$$
n a_{n}=b_{n} .
$$

Consequently, we obtain the following two corollaries.

Corollary 1. If $g \in \mathcal{T}$ is of the Form (36) and its second coefficient $b=b_{2}$ is a fixed real number in $[-2,2]$, then

$$
\left|b_{4}-b_{3}\right| \leq \begin{cases}\frac{7}{4}(2-b) & , b \in[-2,4 / 3] \\ \frac{1}{4}(6-b) & , b \in[4 / 3,2]\end{cases}
$$

Corollary 2. If $g \in \mathcal{T}$ is of the Form (36) and its second coefficient $b=b_{2}$ is a fixed real number in $[-2,2]$, then

$$
\left|b_{n+1}-b_{n}\right| \leq n+1-\frac{1}{2} n b \quad \text { if } n \text { is even }
$$

and

$$
\left|b_{n+1}-b_{n}\right| \leq\left\{\begin{array}{ll}
n+1-\frac{1}{2} n b & , b \in[-2,0] \\
n+1-\frac{1}{2}(n-2) b & , b \in[0,2]
\end{array} \quad \text { if } n \text { is odd } .\right.
$$


Equality holds for the function

$$
\begin{aligned}
g(z) & =\frac{1}{2}\left(1+\frac{p}{2}\right) \frac{z}{(1-z)^{2}}+\frac{1}{2}\left(1-\frac{p}{2}\right) \frac{z}{(1+z)^{2}} \\
& =z+b z^{2}+3 z^{3}+2 b z^{4}+\ldots,
\end{aligned}
$$

if $n$ is even and if $b=-2$ for all positive integers $n$.

\section{Concluding Remarks}

The estimates of various coefficient functionals in the class $\mathcal{C}_{0}(h, p)$ determined in the previous sections were obtained due to connecting this class with the class $\mathcal{P}$ of functions with a positive real part and Lemmas 4 and 5. It is worth noting that all facts about $\mathcal{P}$, including estimates of coefficient functionals, is a good tool in studies of other classes of analytic functions. For this reason, Lemma 6 and 7 obtained in this paper may have independent applications for solving other extreme coefficient problems. It is also worth emphasizing the possibility of obtaining interesting information for functions from classes related to $\mathcal{C}_{0}(h)$, including $\mathcal{K}_{\mathbb{R}}(i)$ and $\mathcal{T}$. It seems that it would be difficult to obtain such results in any other way.

Author Contributions: All authors contributed equally to this work. All authors have read and agreed to the published version of the manuscript.

Funding: The project/research was financed in the framework of the project Lublin University of TechnologyRegional Excellence Initiative, funded by the Polish Ministry of Science and Higher Education (contract no. 030/RID/2018/19).

Conflicts of Interest: The authors declare no conflict of interest.

\section{References}

1. Arora, V.; Ponnusamy, S.; Sahoo, S.K. Successive coefficients for spirallike and related functions. Rev. R. Acad. Cienc. Exactas Fis. Nat. Ser. A Mat. 2019, 113, 2969-2979. [CrossRef]

2. Hamilton, D.H. On a conjecture of M. S. Robertson. J. Lond. Math Soc. 1980, 21, 265-278. [CrossRef]

3. Leung, Y. Successive coefficients of starlike functions. Bull. Lond. Math. Soc. 1978, 10, 193-196. [CrossRef]

4. Li, M. A note on successive coefficients of spirallike functions. Filomat 2018, 32, 1199-1207. [CrossRef]

5. Robertson, M.S. Univalent functions starlike with respect to a boundary point. J. Math. Anal. 1981, 81, 327-345. [CrossRef]

6. Li, M.; Sugawa, T. A note on successive coefficients of convex functions. Comput. Methods Func. Theory 2017, 17, 179-193. [CrossRef]

7. Peng, Z.; Obradović, M. The estimate of the difference of initial successive coefficients of univalent functions. J. Math. Inequal. 2019, 13, 301-314. [CrossRef]

8. Rogosinski, W. Über Positive Harmonische Entwicklungen und Tipisch-Reelle Potenzreichen (German). Math. Z. 1932, 35, 93-121. [CrossRef]

9. Kaplan, W. Close to convex schlicht functions. Mich. Math. J. 1952, 1, 169-185. [CrossRef]

10. Kowalczyk, B.; Lecko, A. The Fekete-Szegö inequality for close-to-convex functions with respect to a certain starlike function dependent on a real parameter. J. Inequal. Appl. 2014, 2014, 65. [CrossRef]

11. Thomas, D.K. The second Hankel determinant of functions convex in one direction. Int. J. Math. Anal. 2016, $10,423-428$.

12. Trąbka-Więcław, K.; Zaprawa, P.; Gregorczyk, M.; Rysak, A. On the Fekete-Szegö type functionals for close-to-convex functions. Symmetry 2019, 11, 1497. [CrossRef]

13. Zaprawa, P. Successive coefficients of close-to-convex functions. Forum Math. 2020. [CrossRef]

14. Zaprawa, P. On the Fekete-Szegö Type Functionals for Functions which Are Convex in the Direction of the Imaginary Axis; Comptes rendus de l'Académie des Sciences: Paris, France, 2020.

15. Robertson, M.S. On the theory of univalent functions. Ann. Math. 1936, 37, 374-408. [CrossRef]

16. Duren, P.L. Univalent Functions; Springer: New York, NY, USA, 1983.

17. Hayami, T.; Owa, S. Generalized Hankel determinant for certain classes. Int. J. Math. Anal. 2010, 4, $2573-2585$. 
18. Libera, R.J.; Złotkiewicz, E.J. Coefficients bounds for the inverse of a function with derivative in P. Proc. Am. Math. Soc. 1983, 87, 251-257. [CrossRef]

19. Libera, R.J.; Złotkiewicz, E.J. Early coefficients of the inverse of a regular convex function. Proc. Am. Math. Soc. 1982, 85, 225-230. [CrossRef]

20. Brown, J.E. Successive coefficients of functions with positive real part. Int. J. Math. Anal. 2010, 4, 2491-2499.

21. Lecko, A. On coefficient inequalities in the Caratheodory class of functions. Ann. Pol. Math. 2000, 75, 59-67. [CrossRef]

22. Choi, J.H.; Kim, Y.C.; Sugawa, T. A general approach to the Fekete-Szegö problem. J. Math. Soc. Jpn. 2007, 59, 707-727. [CrossRef]

Publisher's Note: MDPI stays neutral with regard to jurisdictional claims in published maps and institutional affiliations.

(C) 2020 by the authors. Licensee MDPI, Basel, Switzerland. This article is an open access article distributed under the terms and conditions of the Creative Commons Attribution (CC BY) license (http://creativecommons.org/licenses/by/4.0/). 